\title{
Atypical Cause of Abducens Paralysis: A Case Report
}

\author{
Hikmet Sacmacia, b, Meral Mirza ${ }^{a}$, Mehmet Fatih Yetkin ${ }^{a}$, \\ Ali Ozdemir Ersoy ${ }^{\mathrm{a}}$, Emine Gul Baldane ${ }^{\mathrm{a}}$
}

\begin{abstract}
Isolated cranial nerve paralysis in primary Sjogren's syndrome (SS) has rarely been described. We present a 58-year-old woman who suffered from abducens paralysis and right orbital intractable pain. Cranial MRI findings in right cavernous sinus were associated with granulomatous inflammation. Antinuclear antibody (titre 1/1,000), anti-SS-A, anti-SS-B antibodies were present. SS diagnosis was considered with clinical, laboratory and imaging findings. Immunosuppressive therapy and prednisolone were started. Two months after her initial presentation the patient experienced an almost complete recovery of paralysis of abducens.
\end{abstract}

Keywords: Abducens paralysis; Sjogren's syndrome; Immunosuppressive therapy; MRI

\section{Introduction}

Sjogren's syndrome (SS) is a chronic inflammatory disorder characterized primarily by diminished lacrimal and salivary gland secretions resulting in symptoms of dry eyes and dry mouth [1]. A variety of neurologic symptoms are described in SS. Disease-associated neuropathy is one of the major neurologic complications of SS [2]. Isolated cranial nerve lesions in primary SS, affecting the second, third, sixth and seventh nerves have been reported previously [3].

The main pathogenesis of the neurologic involvement has been reported to be vasculopathy due to cryoglobulinemia and anti-SS-A antibody and ganglionopathy of tri-

Manuscript accepted for publication November 28, 2013

${ }^{a}$ Department of Neurology, Erciyes University, TR-38039, Kayseri, Turkey

${ }^{\mathrm{b} C}$ Corresponding author: Hikmet Sacmaci, Department of Neurology, Erciyes University, 38039 Kayseri, Turkey.

Email: hsacmaci@hotmail.com

doi: http://dx.doi.org/10.4021/jnr247w geminal nerve and dorsal root ganglia. However, the definite pathogenesis has remained unclear. The pathogenesis can be various and complicated [4].

We report a patient presenting with an isolated abducens nerve palsy leading to the diagnosis of SS. The rapid resolution of sixth nerve palsy with immunosuppressive therapy in this patient provides some insights into the pathogenesis of cranial neuropathies in SS.

\section{Case Report}

A 58-year-old female patient was referred to neurology clinic with right retro orbital headache for 2 weeks associated with dropping of the right eyelid, double vision, vomiting and right abducens nerve palsy. There was no history of fever or trauma. But she had right maxillary sinus operation 3 years ago for chronic sinusitis. She was a nonsmoker and non-alcohol drinker. On admission right eye abduction was restricted and there were mild ptosis on right eyelid. Neurologic examination was otherwise normal.

Magnetic resonance imaging (MRI) findings revealed asymmetrical contrast enhancement in right cavernous sinus which was associated with granulomatous inflammation, leading to pachymeningeal thickening (Fig. 1)

Cerebrospinal fluid (CSF) examination was non-traumatic and showed no red cells or leucocytes. CSF protein was $60 \mathrm{mg} / \mathrm{dL}$ and pressure was $6 \mathrm{cmH}_{2} \mathrm{O}$. The CSF was negative for any bacterial growth or neurotropic viruses. Routine blood tests were subsequently performed in view of normal imaging findings. Anti-nuclear antibody (ANA) was found to be positive at $1 / 1,000$ dilution in speckled pattern and anti-SS-A, anti-SS-B antibodies were detected. A diagnosis of SS was considered in view of positive anti-Ro and $\mathrm{La}$ antibodies. In view of positive antibodies, a labial salivary gland biopsy was performed, biopsy revealed nonspecific histopathologic findings. Although salivary gland biopsy did not demonstrate any sign of lymphocytic infiltration, salivary scintigraphy showed evidence of parotid gland involvement. Schirmer's test was positive as $6 \mathrm{~mm}$ of tear flow over $5 \mathrm{~min}$.

The patient began therapy with prednisolone $1 \mathrm{mg} / \mathrm{kg} /$ 

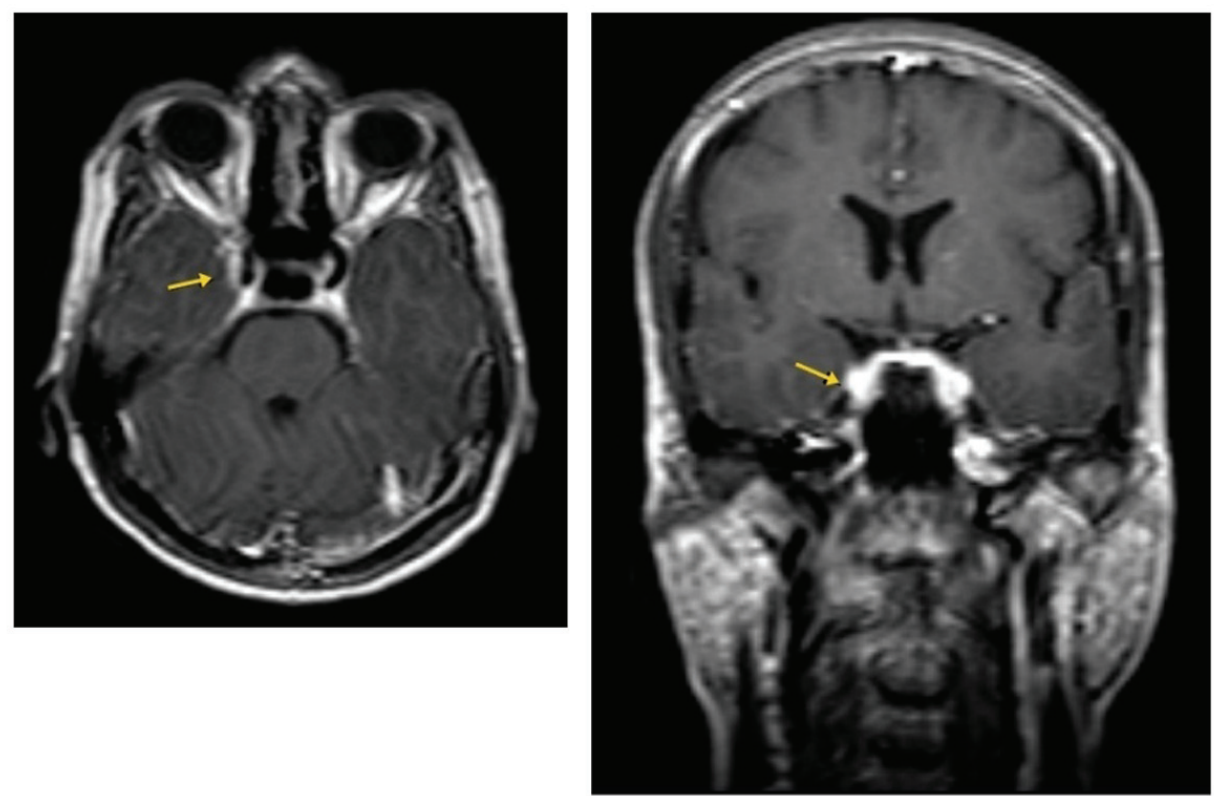

Figure 1. Axial and coronal post-contrast T1 images show asymmetrical contrast enhancement in right cavernous sinus.

day and methotrexate $10 \mathrm{mg} /$ week. Prior to initiation of corticosteroid therapy, there was no improvement in the abducens nerve palsy. After 2 weeks of treatment she experienced an almost recovery of double vision and headache.

\section{Discussion}

This is case report of a patient with isolated reversible sixth nerve palsy due to SS. The frequency of neurologic complications of SS is debated. In previous series, the occurrence of neurologic complications ranged from $12 \%$ (29) to $70 \%$ (39) [4]. Neurologic findings in SS patients include PNS cranial neuropathy, polyneuropathy, mononeuritis multiplex, encephalopathy, aseptic meningitis, cerebral white matter and spinal cord lesions [5]. In a review of neurologic manifestations in 82 patients with SS, $19.5 \%$ of patients had cranial neuropathies while $44.5 \%$ has peripheral neuropathy [6]. Although aseptic meningitis is a common neurologic complication in patients with SS multiple cranial neuropathies are relatively rare [2]. The mechanism for central and peripheral neurological involvement in SS is still unclear. Lymphocytic (T-cell) infiltration of the dorsal ganglia, mononuclear cell infiltration, cryoglobulin-mediated and necrotizing vasculitis, ischemic mechanisms, antineuronal antibodies, a direct role of anti-Ra antibodies have all been suggested as possible causes of the nervous system involvement in SS [1]. Galbussera et al [7] proposed two possible mechanisms related to this cranial neuropathy [8], namely 1) "vascular" (due to damage of the vasa nervorum) or 2) an "immunologic cause" (due to lymphocytic infiltration of the nerve).
The acute presentation of this patient's symptoms suggested that this could be the result of infiltration of cavernous sinus. Improvement of the abducens palsy as well as both MRI and CSF findings after corticosteroid therapy indicated that SS was the main etiology of the cranial neuropathy in this patient. From a radiologic standpoint, lesions of sub cortically (especially periventicular) white matter are more prevalent in patients with SS than in normal control subjects by MRI. Such lesions may be asymptomatic and occur independently of the presence of RF an aCL antibody [9].

In summary for patients with disabling neuropathy who require disease-modifying therapy, in our opinion, the current limited data on immunomodulatory therapy support the first line use of corticosteroids for multiple mononeuropathy and multiple cranial neuropathy and IVIg (intravenous immune globulin) for sensory ataxic and small fiber neuropathy [10].

In conclusion this case illustrates that $\mathrm{SS}$ is rare cause of reversible isolated sixth nerve palsy. Early diagnosis and treatment can reverse the inflammatory process with a good outcome as seen in this patient.

\section{References}

1. Lui NL, See SJ, Thumboo J. A rare cause of reversible unilateral third nerve palsy. J Neurol Sci. 2008;275(12):188-190.

2. Sakai K, Hamaguchi T, Yamada M. Multiple cranial nerve enhancement on MRI in primary Sjogren's syndrome. Intern Med. 2010;49(9):857-859. 
3. Urban PP, Keilmann A, Teichmann EM, Hopf HC. Sensory neuropathy of the trigeminal, glossopharyngeal, and vagal nerves in Sjogren's syndrome. J Neurol Sci. 2001;186(1-2):59-63.

4. Lafitte C, Amoura Z, Cacoub P, Pradat-Diehl P, Picq C, Salachas F, Leger JM, et al. Neurological complications of primary Sjogren's syndrome. J Neurol. 2001;248(7):577-584.

5. Gono T, Kawaguchi Y, Katsumata Y, Takagi K, Tochimoto A, Baba S, Okamoto Y, et al. Clinical manifestations of neurological involvement in primary Sjogren's syndrome. Clin Rheumatol. 2011;30(4):485-490.

6. Delalende S, de Seze J, Fauchias AL, et al. Neurological manifestations of primary Sjogren's syndrome; a study of 82 patients. Medicine (Baltimore). 2004;83:280-291.
7. Galbussera A, Tremolizzo L, Tagliabue E, Ceresa C, Cilia R, Ruffmann C, Ferrarese C, et al. Third cranial nerve palsy? Look for a sicca syndrome. J Neurol Sci. 2007;253(1-2):88-89.

8. Mori K, Iijima M, Koike H, Hattori N, Tanaka F, Watanabe H, Katsuno M, et al. The wide spectrum of clinical manifestations in Sjogren's syndrome-associated neuropathy. Brain. 2005;128(Pt 11):2518-2534.

9. Anaya JM, Villa LA, Restrepo L, Molina JF, Mantilla RD, Vargas S. Central nervous system compromise in primary Sjogren's syndrome. J Clin Rheumatol. 2002;8(4):189-196.

10. Chai J, Logigian EL. Neurological manifestations of primary Sjogren's syndrome. Curr Opin Neurol. 2010;23(5):509-513. 